\title{
Demo Abstract: E-Eye: mmWave Nonlinear Response for Hidden Electronic Device Recognition
}

\author{
Baicheng Chen ${ }^{1}$, Zhengxiong $\mathrm{Li}^{1}$, Zhuolin Yang ${ }^{1}$, Changzhi $\mathrm{Li}^{2}$, Feng $\operatorname{Lin}^{3}$ and Wenyao $\mathrm{Xu}^{1}$ \\ ${ }^{1}$ University at Buffalo, the State University of New York, Buffalo, New York, USA \\ ${ }^{2}$ Texas Tech University, Lubbock, Texas, USA \\ ${ }^{3}$ Zhejiang University, Hangzhou, Zhejiang, China \\ \{baicheng,zhengxio,zhuoliny,wenyaoxu\}@buffalo.edu,changzhi.li@ttu.edu,flin@zju.edu.cn
}

\begin{abstract}
Hidden electronics possess the risk of both security threat and privacy intrusion. We present a wireless hidden electronic recognition system, through electronic components unique mmWave nonlinear responses to identify the threats. We then evaluate E-Eye's performance and robustness with a controlled experiment and a field study using iconic devices and score the system with metrics. Results prove that E-Eye is an accurate and robust hidden electronic recognition system.
\end{abstract}

\section{INTRODUCTION}

This demo is based on our published work [4]. Countless security and privacy breaches from hidden electronic devices have caused both financial and sociological problems $[1,2]$. From hidden camera and microphone eavesdropping in private zones to remote weapons in camouflage causing deaths and injuries, brutal lessons urge the need to recognize hidden e-devices before more victims are harmed. Current methods utilize security gate at the entrance to detect and eliminate malicious objects such as X-ray detector, metal detector, and Infrared detector are limited in recognition ability, application feasibility, and personal safety [7].

E-Eye is based on electronic devices (e-devices) emitting a unique nonlinear response at the interrogation of mmWave. As shown in Fig. 3, E-Eye starts with transmitting interrogation signal from the Tx terminal of the mmWave probe, as the signal reaches each e-devices, a nonlinear response is generated from each of the devices. Once the nonlinear response is received at the $\mathrm{Rx}$ terminal of mmWave probe, recognition of hidden e-devices is performed.

The crux of E-Eye is the nonlinear response of each e-devices, which bases on the unique pattern of electronic components in their printed circuit boards (PCB). Each of the electronic components of the PCB contributes to the nonlinear response by reflecting the interrogation signal, this results in a response signal that describes the pattern in the form of electromagnetic wave. Such reflection property of e-devices serves as their descriptor, which definitively

The video link: "https://youtu.be/apgRH3cDKdQ".

Permission to make digital or hard copies of all or part of this work for personal or classroom use is granted without fee provided that copies are not made or distributed for profit or commercial advantage and that copies bear this notice and the full citation on the first page. Copyrights for components of this work owned by others than ACM must be honored. Abstracting with credit is permitted. To copy otherwise, or republish, to post on servers or to redistribute to lists, requires prior specific permission and/or a fee. Request permissions from permissions@acm.org.

SenSys '19, November 10-13, 2019, New York, NY, USA

(c) 2019 ACM.

ACM ISBN 978-1-4503-6950-3/19/11_..\$15.00

https://doi.org/10.1145/3356250.3361932 grants their signal modulation to be unique. The modulated signal is reflected to mmWave probe from the e-devices, allows recognition infer the responding e-devices.

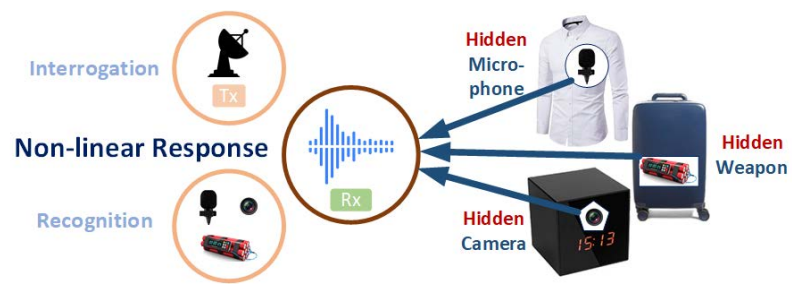

Figure 1: E-Eye's hidden electronic recognition examples.

\section{SYSTEM DESIGN}

The system accomplishes hidden e-devices recognition through four stages, nonlinear response acquisition, signal-wavelet transform, feature extraction, and device classification.

Nonlinear Response Acquisition: We implement the $24 \mathrm{GHz}$ mmWave radar that accommodates typical trace length of PCB to capture the unique nonlinear response from e-devices. As shown in Fig. 2, the radar probe sends interrogation signal through its Tx port, and collects response signal with its Rx port.

Wavelet Transform: We implement wavelet transform of nonlinear response signal to decompose it into sub-bands for better analysis. The reconstruction of nonlinear response from the collection of wavelet functions is formulated by Eq. (1):

$$
\begin{aligned}
\underbrace{y(t)}_{\text {Nonlinear response }}= & \underbrace{\frac{1}{C_{\phi}} \int_{-\infty}^{\infty} F_{W}\left(a_{0}, b\right) \phi_{a_{0}, b} \frac{d b}{\sqrt{a_{0}}}}_{\text {The approximation part }} \\
& \underbrace{\frac{1}{C_{\psi}} \int_{a_{1}}^{\infty} \int_{-\infty}^{\infty} F_{W}(a, b) \psi_{a, b} \frac{d a}{a^{2}} \frac{d b}{\sqrt{a}}}_{\text {The detail part }},
\end{aligned}
$$

where $\mathrm{t}$ is time, $y(t)$ is the nonlinear response in spectral-temporal domain from electronic device [6]. $F_{W}(a, b)$ is the time-scale map coefficients, $a$ and $b$ are the scale and translation parameters. $\phi_{a, b}$ and $\psi_{a, b}$ are the mother wavelet functions for approximation part and detailed part that utilizes $C_{\phi}$ and $C_{\psi}$ constants, respectively. Feature Extraction: We implement 13 scalar features in spectraltemporal domains to characterize each nonlinear response. The spectral features are responsible for spectral-frequency related characteristics of a wavelet, whereas the temporal features are responsible for property in an amplitude-time relationship as shown in Table 1. In addition, we adopt 10 Mel-Frequency Cepstral Coefficients 


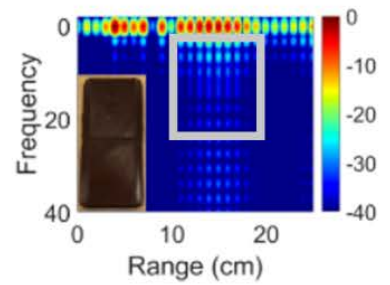

3D Printed Object

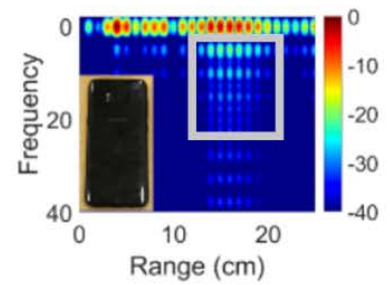

Galaxy $\mathrm{s} 8$

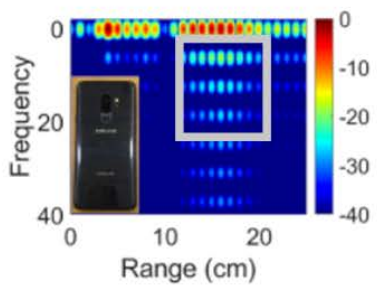

Galaxy s9+

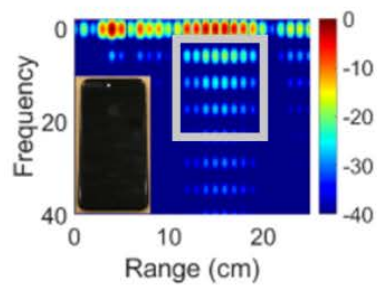

Iphone 7p

Figure 2: Difference in nonlinear response from three typical e-devices and one 3D printed object in spectral analysis.

Table 1: Feature List for nonlinear response recognition

\begin{tabular}{c||c}
\hline Domain & Features \\
\hline Spectral & $\begin{array}{c}\text { Flatness, Crest Factor, Skewness, Kurtosis, Mean } \\
\text { Value, Standard Deviation (STD), MFC-10 }\end{array}$ \\
\hline Temporal & $\begin{array}{c}\text { Skewness, Kurtosis, RMS Amplitude, Lowest } \\
\text { Value, Mean Value, Highest Value, STD }\end{array}$ \\
\hline
\end{tabular}

(MFCC-10) which describes the nonlinear response in auditory field. The total of 46 features, with 23 for approximation part and 23 for detail part enables accurate recognition of hidden e-devices.

Hidden Electronic Recognition: We label each electronic device by their extracting their features and fit the feature data into a Support Vector Machine (SVM) for accurate classification. To train the SVM, we use a Gaussian radial basis function kernel to fit the extracted features. After the model is established, recognition of an electronic device is done within few milliseconds using the prediction function.

\section{EVALUATION}

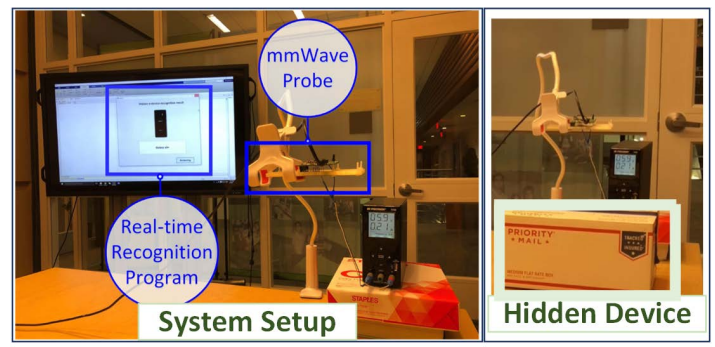

Figure 3: Device Setup for sensing.

As the development of $5 \mathrm{G}$ network scales, mmWave radar is also used toward identification in many scenarios $[3,5,8]$. We utilize a 24 $\mathrm{GHz}$ mmWave radar with industrial, scientific, medical (ISM) band. The electronic devices are placed in cloth sleeves, paper packaging, plastic wrap, or obstructed with 1-inch wood piece for interference. We utilize 40 devices from multiple groups of electronic devices to perform inter-group classification and intra-group classification We display the recognition result with confusion matricies as shown in Fig. 4. The result collected from the model prediction of 40 devices with 100 samples from each device. The percentage accuracy of the classification was $99.9 \%$ for both inter-group and intra-group classification. Result proves that E-Eye's capability in accurately recognizing hidden electronic devices. To display the process of hidden electronic device recognition, we prepared a demo video (video link attached).

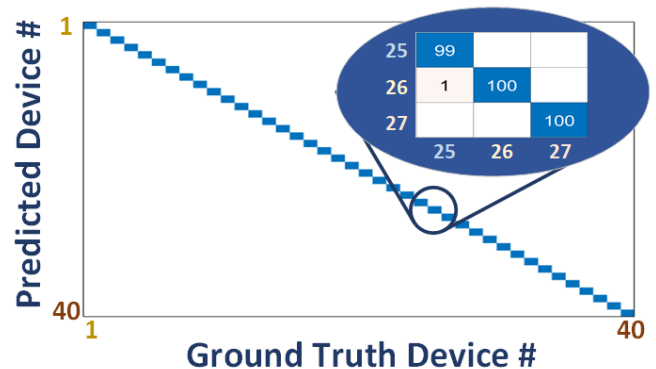

Figure 4: Confusion matrix for prediction result of 40 electronic devices. Deeper color represents higher occurrence.

\section{CONCLUSION}

Our work examines and confirms the recognition of hidden electronic devices using unique nonlinear response. Our developed system allows hidden device recognition with various occlusion and interference. The technology can be applied widely due to its low-cost and non-human harming nature.

\section{ACKNOWLEDGEMENT}

We thank our anonymous reviewers for their insightful feedback. This work was in part supported by the National Science Foundation under grant No. 1718375/1718483.

\section{REFERENCES}

[1] Douglass Dowty. Geddes town supervisor's secretary faces felony for eavesdropping on co-workers, Dec 2016.

[2] Jon Gambrell. Bombs disguised as rocks in yemen reportedly show iran aid, Mar 2018.

[3] Zhengxiong Li, Fenglong Ma, Aditya Singh Rathore, Zhuolin Yang, Baicheng Chen, $\mathrm{Lu}$ Su, and Wenyao Xu. Wavespy: Remote and through-wall screen attack via mmwave sensing. In To appear in IEEE Symposium on Security and Privacy 2020, SP'20, 2020.

[4] Zhengxiong Li, Zhuolin Yang, Chen Song, Changzhi Li, Zhengyu Peng, and Wenyao Xu. E-eye: Hidden electronics recognition through mmwave nonlinear effects. In Proceedings of the 16th ACM Conference on Embedded Networked Sensor Systems, pages 68-81. ACM, 2018.

[5] Feng Lin, Chen Song, Yan Zhuang, Wenyao Xu, Changzhi Li, and Kui Ren. Cardiac scan: A non-contact and continuous heart-based user authentication system. In Proceedings of the 23rd Annual International Conference on Mobile Computing and Networking, pages 315-328. ACM, 2017.

[6] Satish Sinha, Partha S Routh, Phil D Anno, and John P Castagna. Spectral decomposition of seismic data with continuous-wavelet transform. Geophysics, 70(6):P19-P25, 2005.

[7] Colin Stagner. Detecting and locating electronic devices using their unintended electromagnetic emissions. 2013.

[8] Chenhan Xu, Zhengxiong Li, Hanbin Zhang, Aditya Singh Rathore, Huining Li, Chen Song, Kun Wang, and Wenyao Xu. Waveear: Exploring a mmwave-based noise-resistant speech sensing for voice-user interface. In Proceedings of the 17th Annual International Conference on Mobile Systems, Applications, and Services, pages 14-26. ACM, 2019. 